\title{
C-Fos Digital Expression Analysis in Human Papillomavirus- Related Oral Squamous Cell Carcinoma
}

\author{
Aristeidis Chrysovergis ${ }^{1}$, Vasileios Papanikolaou ${ }^{1}$, Nicholas Mastronikolis ${ }^{2}$, \\ Despoina Spyropoulou ${ }^{3}$, Maria Adamopoulou ${ }^{4}$, Evangelos Tsiambas ${ }^{5}$, Vasileios \\ Ragos $^{6}$, Dimitrios Peschos ${ }^{7}$, Dimitrios Roukas ${ }^{8}$, Chara Stavraka ${ }^{9}$, Athanasios \\ Niotis $^{10}$, Efthymios Kyrodimos ${ }^{1}$
}

${ }^{1}$ 1st ENT Department, Hippocration Hospital, University of Athens, Athens, Greece. ${ }^{2}$ ENT Dept, Medical School, University of Patras, Greece. ${ }^{3}$ Department of Radiation Oncology, Medical School, University of Patras, Greece. ${ }^{4}$ Department of Biomedical Sciences, University of West Attica, Athens, Greece. ${ }^{5}$ Department of Cytology, 417 VA Hospital (NIMTS), Athens, Greece, ${ }^{6}$ Department of Maxillofacial, Medical School, University of Ioannina, Greece. ${ }^{7}$ Department of Physiology, Medical School, University of Ioannina, Greece. ${ }^{8}$ Department of Psychiatry, 417 VA Hospital (NIMTS), Athens, Greece. ${ }^{9}$ Department of Medical Oncology, Guy's and St Thomas NHS Foundation Trust, London, UK. ${ }^{10}$ Department of Surgery, 417 VA Hospital (NIMTS), Athens, Greece.

\begin{abstract}
Background: Fos Proto-Oncogene (c-Fos) represents a well analyzed gene involved in solid malignancies' development and progression. The corresponding protein forms heterodimer with c-jun, a strong transcription factor. C-Fos/c-Jun complex influences critically the intracellular signal transduction to the nucleus. Our aim was to detect and evaluate c-Fos protein expression patterns in oral squamous cell carcinomas (OSCC) tissues. Materials and Methods: Fifty $(\mathrm{n}=50)$ formalin-fixed, paraffin-embedded primary OSCCs tissue sections were used. Immunohistochemistry and digital image analysis were implemented for identifying and evaluating c-Fos protein expression levels, respectively. Results: C-Fos protein over expression (moderate to high imunostaining intensity values) was observed in 28/50 (56\%) tissue cores, whereas low expression rates were detected in the rest of the examined cases $(22 / 50-44 \%)$. C-Fos overall expression was strongly associated with the stage and grade of the examined tumors $(\mathrm{p}=0.014, \mathrm{p}=0.003$, respectively) and also with Human papillomavirus (HPV) persistent infection $(\mathrm{p}=0.004)$. c-Fos up regulation is frequently observed in OSCCs. Conclusion: C-Fos high expression levels are correlated with an aggressive phenotype (advanced stage/lymph node metastasis) in patients with OSCC, especially in HPV positive cases, especially High Risk subtypes. Due to its elevated oncogenic activity, c-Fos should be a target for novel therapeutic strategies in OSCC combined or not with other oncogenes involving in signaling transduction pathways.
\end{abstract}

Keywords: Carcinoma- oral- c-Fos- gene- image analysis- immunohistochemistry

\section{Introduction}

Solid malignancies demonstrate molecular profiles based on oncogenes' over activation combined with suppressor genes silence. Concerning oncogenes, Fos Proto- Oncogene or AP-1 Transcription Factor Subunit (c-Fos) represents a critical gene involved in a variety of malignant tumours' development and progression, including oral squamous cell carcinoma
(OSCC). The Fos superfamily comprises c-Fos, FosB, FosL1, and FosL2 genes. c-Fos is a proto-oncogene that is the human homolog of the retroviral oncogene v-fos (gene locus: 14q24.3). It was initially analyzed and cloned in rat fibroblasts as the transforming gene of Finkel-Biskis-Jinkins murine osteogenic sarcoma virus [1]. The gene encodes a $62 \mathrm{kDa}$ protein (380 amino acids),

Corresponding Author:

Dr. Evangelos Tsiambas

Department of Cytology, 417 VA Hospital (NIMTS), Athens, Greece.

Email: tsiambasecyto@yahoo.gr 
forming heterodimer with c-jun, a strong transcription factors), resulting in the formation of AP-1 (Activator Protein-1) complex. C-Fos/c-Jun complex influences intracellular signal transduction to the nucleus. c-Fos protein is implicated in critical cell functions including differentiation, proliferation, survival and also tissue homeostasis affected by hypoxia and angiogenesis [2]. Concerning OSCC, c-Fos aberrant expression seems to be a frequent event, especially in sub groups of patients associated on not with their corresponding clinico-histological features [3-4]. In the current study, we analyzed c- Fos protein expression levels in OSCC tissue sections by implementing a digital image analysis protocol on immunostined slides. To our knowledge there are very limited published data regarding the current methodology in OSCC oncoproteins' analyses.

\section{Materials and Methods}

\section{Study group}

For the purposes of our study, fifty $(n=50)$ archival, formalin-fixed and paraffin-embedded tissue specimens of histologically confirmed primary OSCC were used. Selection of the cases was based predominantly on the criterion of an aggressive phenotype as it is expressed due to advanced Grade and Stage histo-pathological features. The hospital ethics committee consented (Reference ID Research Protocol: 2226/09.09.2018) to the use of these tissues for research purposes, according to World Medical Association Declaration of Helsinki guidelines (2008, revised 2014). The tissue samples were fixed in $10 \%$ neutral-buffered formalin. Hematoxylin and eosin (H\&E)-stained slides of the corresponding samples were reviewed for confirmation of histopathological diagnoses. All lesions were classified according to the histological typing criteria of World Health Organization (WHO) (5). Concerning HPV DNA status (positivity or not), the corresponding information was derived from patients' medical file records. Among them, $18 \mathrm{HPV}$ DNA positive cases were recorded. HPV 16/31/53 High Risk (HR) subtypes were detected mainly by analyzing the corresponding cases. Clinicopathological data of the examined cases are demonstrated in Table 1.

\section{Antibodies and immunohistochemistry assay (IHC)}

For the purposes of our study, we selected and applied the mouse monoclonal anti- c-Fos mouse monoclonal (clone CF2, Novocastra, Leica Biosystems, Newcastle, $\mathrm{UK}$; dilution 1:40). IHC protocol for the antigen detection was carried out on a $4 \mu \mathrm{m}$ thick paraffin sections of the current blocks. Tissue sections initially deparaffinized in xylene and rehydrated via graded ethanol - was immunostained according to the EN Vision + (DAKO, Denmark) assay using an automated staining system (I 6000 - Biogenex, CA, USA) and according to the corresponding antibodies manufacturer's instructions. This specific assay is based on a soluble, dextranpolymer system preventing endogenous biotin reaction and increasing the quality of the stained slides. Briefly, the sections, after peroxidase blocking, were incubated with primary antibody for $30 \mathrm{~min}$ at room temperature and then incubated with Horseradish peroxidise labeled polymer-HRP LP for $30 \mathrm{~min}$. A wash with TBS was performed. The antigen - antibody reaction was visualized using 3-3, diaminobenzidine tetrahydrocloride (DAB) as a chromogen substrate ( $8 \mathrm{~min}$ at room temperature). Finally, the tissue sections were slightly counterstained with hematoxylin for $30 \mathrm{sec}$, dehydrated and mounted. For negative control slides, the primary antibody was omitted. Nuclear predominantly but also peri-nuclear/cytoplasmic staining pattern was considered to be acceptable for the marker. Normal (non-cancerous) skin tissue sections demonstrating c-Fos expression was used as positive markers for its immunostaining pattern (Figure 1a,b).

\section{Digital Image Analysis assay (DIA)}

C-fos protein expression levels were evaluated quantitatively by calculating the corresponding staining intensity levels (densitometry evaluation) in the immunostained malignant cells. We performed DIA using a semi-automated system (hardware: Microscope CX-31, Olympus, Melville, NY, USA, Digital camera, Sony, Tokyo, Jp; Windows XP/NIS-Elements Software AR v3.0, Nikon Corp, Tokyo, Japan). Areas of interest per tissue section were identified ( 5 optical fields at $\times 400$ magnification) and filed in a digital database as snapshots. Measurements were performed by implementing a specific macro (mainly nuclear for tumor cells, according to manufacturer's datasheet for monoclonal mouse anti-cfos, Clone CF2, Novocastra, Leica Biosystems Newcastle Ltd, UK). Based on an algorithm, normal tissue sections (control) were measured independently and compared to the corresponding values in malignant tissue sections. A broad spectrum of continuous grey scale values (0-255) at the RedGreenBlue (RGB) analysis was available for detecting and discriminating different protein expression levels (Figure 2). Immunostaining intensity values decreasing to 0 represent a progressive over expression of the marker, whereas values increasing to 255 show a progressive loss of its staining intensity. Total results and DIA values are demonstrated in Table 1 .

\section{Statistical analysis}

Statistics software package IBM SPSS v25 (SPSS Inc, Chicago, IL, USA) was implemented. Associations between variables were assessed with of Pearson Chi- Square $(\chi 2)$ test and Fisher's exact. Correlation analysis with Spearman Rank test was performed for variables with significant chi2 associations. Two-tailed p-values $\leq 0.05$ were considered statistically significant. Results and correlations ( $p$-values) are described in Table 1.

\section{Results}

According to DIA implementation, all of the examined cases demonstrated c- Fos expression in different levels. C-Fos protein over expression (moderate to high imunostaining intensity values) was observed in $28 / 50$ $(56 \%)$ tissue sections, whereas low expression rates were 
Table 1. Clinicopathological Parameters and Total c-Fos Expression Results

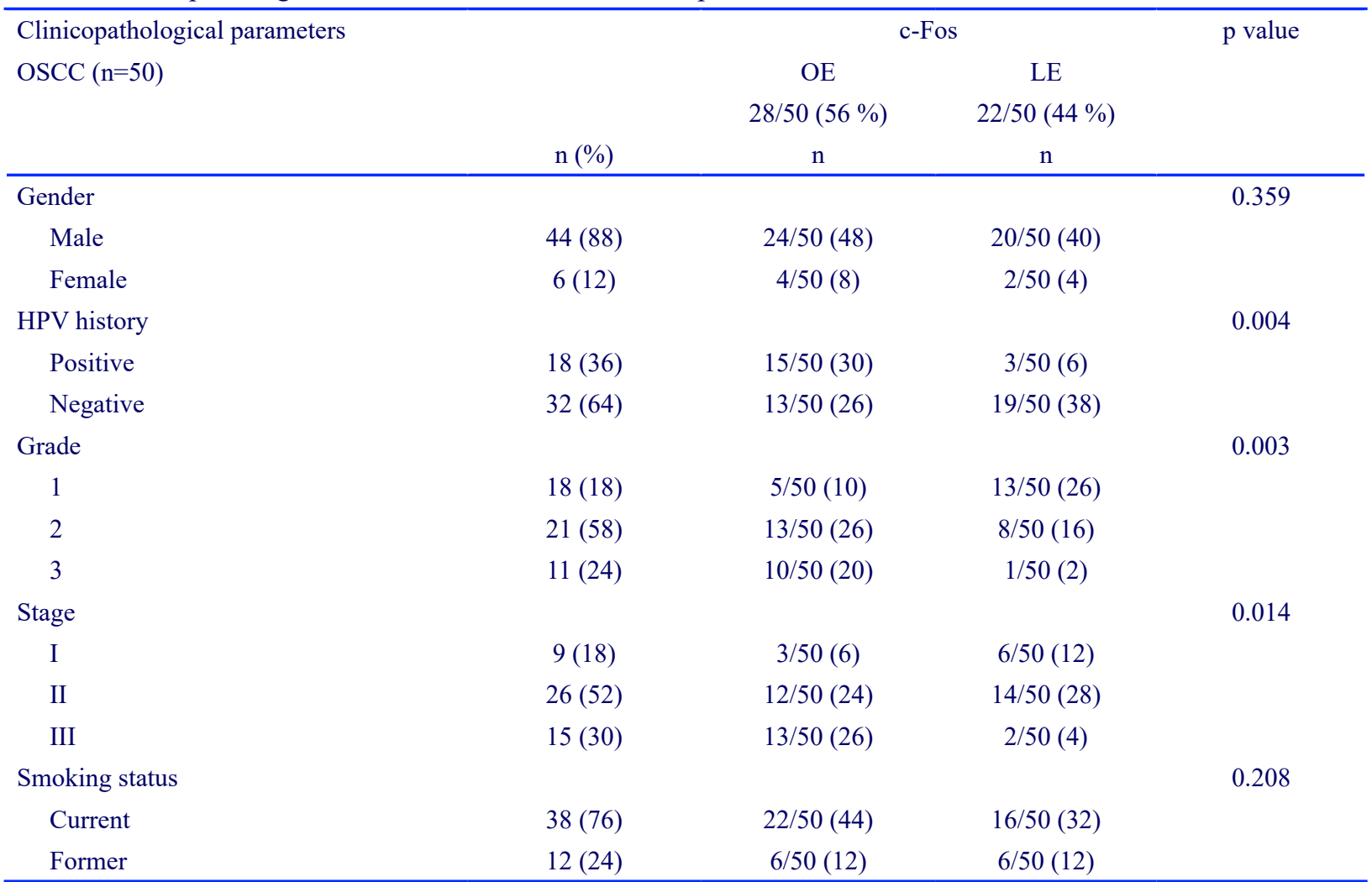

OSCC, oral squamous cell carcinomas; OE, overexpression (moderate to high expression) staining intensity; values $\leq 142$ on the examined stained nuclei; LE, low expression staining intensity values $>156$ on the examined stained nuclei

\section{Discussion}

detected in the rest of the examined cases (22/50-44\%). C-Fos protein over expression (moderate to high imunostaining intensity values) was observed in $28 / 50$ $(56 \%)$ tissue cores, whereas low expression rates were detected in the rest of the examined cases (22/50-44\%). C-Fos overall expression was strongly associated with the stage and grade of the examined tumors $(\mathrm{p}=0.014$, $\mathrm{p}=0.003$, respectively) and also with HPV persistent infection $(p=0.004)$. No other statistical correlations were identified regarding the other clinic-pathological parameters (gender: $\mathrm{p}=0.359$, smoking status: $\mathrm{p}=0.208$ ).
Oral cavity carcinoma represents a major proportion of Head and Neck Squamous Cell Carcinoma (HNSCC). OSCCs demonstrate an aggressive phenotype due to their increased capability to locally metastasize combined with distant lymph node metastases [6]. In solid malignancies deregulated transcriptional factors are correlated with aberrant gene expression [7]. Concerning OSCC, c-Fos over activation seems to be associated with other transcription factors (c-Jun/Fra-1) deregulation [8-9]. In fact, their high co-expression is correlated with poor prognosis in the corresponding patients. Besides

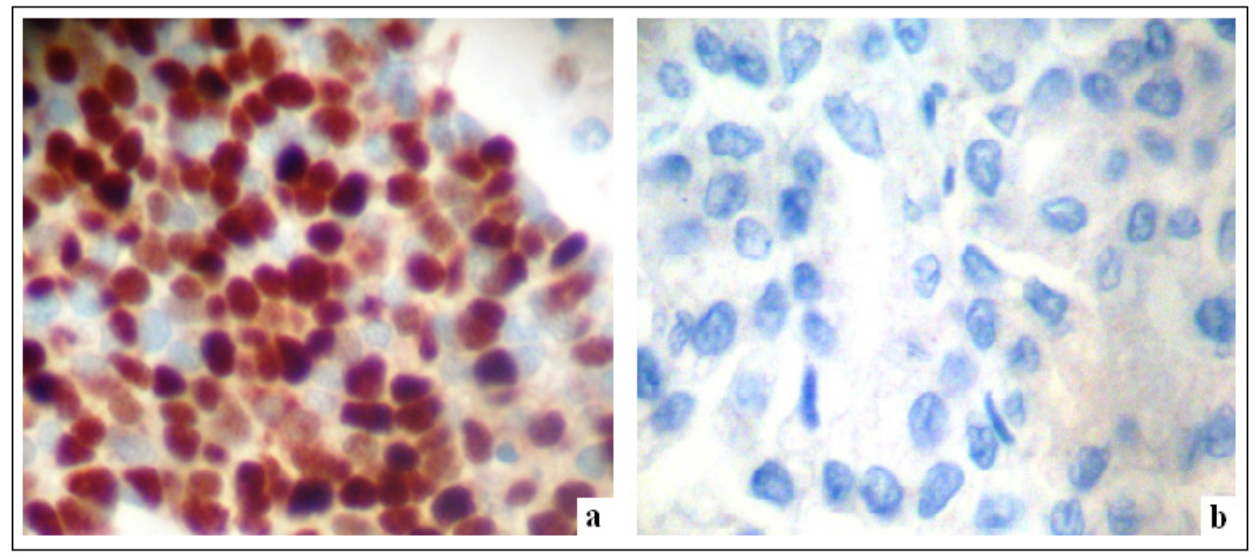

Figure 1. C-Fos Expression Patterns on OSCC Tissue Sections. a. Over expression of c-Fos. Note nuclear mainly dense brown staining pattern (DAB stain, original magnification: 400x) b. Loss of expression (DAB stain, original magnification: 400x) 


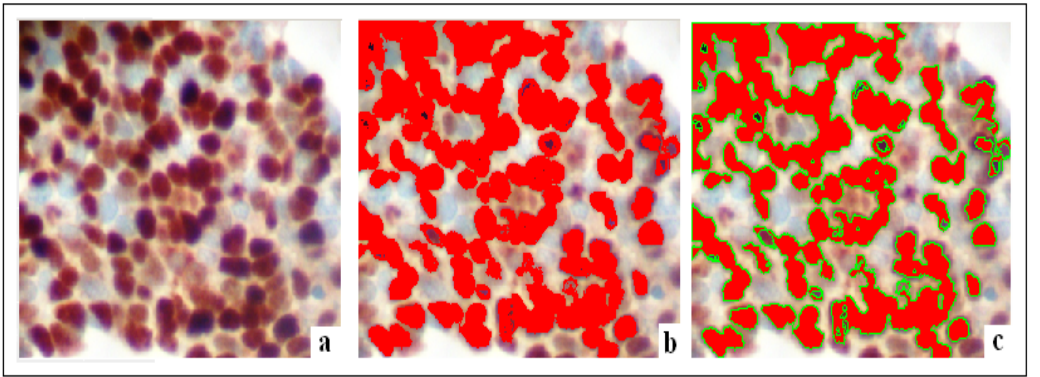

Figure 2. C-Fos Progressive Digital Image Analysis on OSCC Tissue Sections. a. Over expression of c-Fos. Note nuclear mainly and peri-nuclear/cytoplasmic brown staining pattern (DAB stain, original magnification: 100x) b. Red spots represent different expression values of c-Fos expression c. Green loops surrounding red spots represent the final stage of digital analysis providing numerical data (staining intensity values -DAB stain, original magnification: 100x)

these transcriptional factors, the role of c-Fos/mutant p53 protein in OSCC is under investigation. A study group analyzed their coexpression exploring also the involvement of another nucleolar protein (nucleophosmin) in their rise and progression [10]. They reported that high c-Fos/p53 expression levels lead to nucleophosmin up-regulation as a potential synergetic action of these molecules.

In the current study, we analyzed c-Fos protein expression by IHC on OSCC tissue sections measuring the levels of its staining intensity by implementing a DIA protocol. C-Fos up regulation (moderate to high nuclear/peri-nuclear cytoplasmic protein expression levels) was detected in a significant proportion of the examined cases. Overall protein expression was found to be correlated with the stage and also with HPV positivity. Concerning HPV-mediated carcinogenesis in oral mucosa, some molecular studies detected specific HPV-positive signatures that affect signal transduction pathways. In one of them, the role of HPV persistent infection in activation of AP-1, NF- $\kappa$ B, and STAT3 genes was explored. The study group concluded that there is a potential involvement of them in HPV-positive OSCC leading also to c-Fos aberrant expression [11]. Similarly, another experimental cell culture-based study focused on the influence of HPV16 E6 oncogene in c-Fos deregulation. The researchers reported that besides AP-1, c-Fos protein expression is increased by TGF- $\alpha$ in HPV16E6 positive cases [12]. Involvement of growth factors, such as TGF-a in HPV-dependent OSCC should lead to new therapeutic approaches in subsets of patients characterized by specific gene signatures. Similarly, induced apoptotic activity in OSCC is a major issue for research. According to apoptosis analyses in these malignancies, a study suggested that HOX-PBX complex inhibition by a specific agent (HXR9 peptide) is correlated also with c-Fos up regulation [13]. Furthermore, co-analysis of c-Fos/cJun, and also p53 immunohistochemistry led to a significant association with lymph node metastasis, poor differentiation and clinical stage of the examined OSCC tissues. The study group suggested this co-expression as a potential independent prognostic factor for overall survival in the corresponding patients [14].

In conjunction to the previously described results, another study detected over activation of c-Fos in invasive
OSCC compared to adjacent non-malignant epithelia. A combination of nuclear and peri-nuclear cytoplasmic diffuse immunostaining was observed especially in cases demonstrated lymph node metastasis implicating also CD44-depended signal transduction pathway in patients with advanced stage [15]. Additionally, the role of another signal transduction pathway (Notch) in c-Fos over activation is under investigation. A study group showed that targeting overexpressed Notch genes - including JAG1/JAG2- in OSCC by applying $\gamma$-secretase molecule, c-Fos m RNA levels were also decreased [16]. Novel molecular technologies in OSCC gene screening based on c-DNAs microarrays have also detected distinct patterns of gene expression in different sub-group of patients, including oncogenes [17]. Concerning c-fos protein expression evaluation, we implemented a DIA protocol that provides an objective, accurate and fast mean for measuring the immunostaining intensity levels. According to our published experience in a variety of biomarkers, DIA is a useful tool for research and diagnostic reasons -under specific terms- enhancing the innovative evidencebased medicine [18-22].

In summary, c-Fos is a critical gene frequently up regulated in OSCCs. C-Fos high expression levels are correlated with an aggressive phenotype (advanced stage/ lymph node metastasis) in patients with OSCC. HR HPVmediated carcinogenesis in a subset of cases is associated also with increased c-Fos protein expression. Due to its elevated oncogenic activity, c-Fos should be a target for novel therapeutic strategies in OSCC combined or not with other oncogenes involving in signalling transduction pathways.

\section{Acknowledgments}

Authors acknowledge the significant scientific contribution of George Vilaras, technologist in the Department of Pathology, Medical School, University of Athens, Greece, as an expert in IHC/ICC/CISH techniques.

Sources of funding

None. 


\section{Declaration of competing interest}

None.

\section{References}

1. Curran T, Peters G, Van Beveren C, Teich NM, Verma IM. FBJ murine osteosarcoma virus: identification and molecular cloning of biologically active proviral DNA.. Journal of Virology. 1982;44(2):674-682. https://doi.org/10.1128/ jvi.44.2.674-682.1982

2. Turatti E, da Costa Neves A, de Magalhães MHCG, de Sousa SOM. Assessment of c-Jun, c-Fos and cyclin D1 in premalignant and malignant oral lesions. Journal of Oral Science. 2005;47(2):71-76. https://doi.org/10.2334/ josnusd.47.71

3. Krishna A, Bhatt MLB, Singh V, Singh S, Gangwar PK, Singh US, Kumar V, Mehrotra D. Differential Expression of c-fos Proto-Oncogene in Normal Oral Mucosa versus Squamous Cell Carcinoma. Asian Pacific Journal of Cancer Prevention. 2018 03;19(3). https://doi.org/10.22034/ APJCP.2018.19.3.867

4. Mishra A, Bharti AC, Saluja D, Das BC. Transactivation and expression patterns of Jun and Fos/AP-1 super-family proteins in human oral cancer. International Journal of Cancer. 2009;:NA-NA. https://doi.org/10.1002/ijc.24807

5. El-Naggar A, Chan J, Grandis J, et al. World health organization classification of head and neck tumours. 4th ed. Lyon: IARC Press. 2017.

6. Ali J, Sabiha B, Jan HU, Haider SA, Khan AA, Ali SS. Genetic etiology of oral cancer. Oral Oncology. 2017 07;70:23-28. https://doi.org/10.1016/j.oraloncology.2017.05.004

7. Hanahan D, Weinberg R. Hallmarks of Cancer: The Next Generation. Cell. 2011 03;144(5):646-674. https://doi. org/10.1016/j.cell.2011.02.013

8. Xu H, Jin X, Yuan Y, Deng P, Jiang L, Zeng X, Li X, Wang $Z$, Chen Q. Prognostic value from integrative analysis of transcription factors c-Jun and Fra-1 in oral squamous cell carcinoma: a multicenter cohort study. Scientific Reports. 201708 08;7(1). https://doi.org/10.1038/s41598-01705106-5

9. Gupta S, Kumar P, Kaur H, Sharma N, Saluja D, Bharti AC, Das BC. Selective participation of c-Jun with Fra-2/c-Fos promotes aggressive tumor phenotypes and poor prognosis in tongue cancer. Scientific Reports. 2015 Nov 19;5(1). https:// doi.org/10.1038/srep16811

10. Senapati P, Dey S, Sudarshan D, Das S, Kumar M, Kaypee S, Mohiyuddin A, Kodaganur GS, Kundu TK. Oncogene c-fos and mutant $\mathrm{R} 175 \mathrm{H}$ p53 regulate expression of Nucleophosmin implicating cancer manifestation. The FEBS Journal. 201808 24;285(18):3503-3524. https://doi. org/10.1111/febs. 14625

11. Verma G, Vishnoi K, Tyagi A, Jadli M, Singh T, Goel A, Sharma A, Agarwal K, Prasad SC, Pandey D, Sharma S, Mehrotra R, Singh SM, Bharti AC. Characterization of key transcription factors as molecular signatures of HPV-positive and HPV-negative oral cancers. Cancer Medicine. 201702 03;6(3):591-604. https://doi.org/10.1002/cam4.983

12. Liang F, Kina S, Takemoto H, Matayoshi A, Phonaphonh T, Sunagawa N, Arakaki K, Arasaki A, Kuang H, Sunakawa H. HPV16E6-Dependent c-Fos Expression Contributes to AP-1 Complex Formation in SiHa Cells. Mediators of Inflammation. 2011;2011:1-7. https://doi. org $/ 10.1155 / 2011 / 263216$

13. Platais C, Radhakrishnan R, Niklander Ebensperger S, Morgan R, Lambert DW, Hunter KD. Targeting HOX-PBX interactions causes death in oral potentially malignant and squamous carcinoma cells but not normal oral keratinocytes. BMC Cancer. 201807 06;18(1). https://doi.org/10.1186/ s12885-018-4622-0

14. Wang S, Xu X, Xu F, Meng Y, Sun C, Shi L, Zhao E. Combined Expression of c-jun, c-fos, and p53 Improves Estimation of Prognosis in Oral Squamous Cell Carcinoma. Cancer Investigation. 201608 24;34(8):393-400. https://doi. org/10.1080/07357907.2016.1217422

15. Dong C, Ye D, Zhang W, Pan H, Zhang Z, Zhang L. Overexpression of c-fos promotes cell invasion and migration via CD44 pathway in oral squamous cell carcinoma. Journal of Oral Pathology \& Medicine. 2014 Dec 08;44(5):353-360. https://doi.org/10.1111/jop.12296

16. Osathanon T, Nowwarote N, Pavasant P. Expression and influence of Notch signaling in oral squamous cell carcinoma. Journal of Oral Science. 2016;58(2):283-294. https://doi.org/10.2334/josnusd.15-0535

17. Lunde M, Warnakulasuriya S, Sand L, et al. Gene expression analysis by cDNA microarray in oral cancers from two Western populations. Anticancer Res. 2010;30:1083-91.

18. Chrysovergis A, Papanikolaou Vs, Tsiambas E, Ragos V, Peschos D, Kyrodimos E. Digital Analysis of BCL2 Expression in Laryngeal Squamous Cell Carcinoma. Anticancer Research. 2019 03;39(3):1253-1257. https://doi. org/10.21873/anticanres. 13235

19. Papadas T, Tsiambas E, Mastronikolis N, et al. Topoisomerase I deregulation in laryngeal squamous cell carcinomas based on tissue microarray analysis. JBUON. 2017;22:771-6.

20. Politi A, Tsiambas E, Mastronikolis Ns, Peschos D, Asproudis I, Kyrodimos E, Armata Ie, Chrysovergis A, Asimakopoulos A, Papanikolaou Vs, Batistatou A, Ragos V. Combined EGFR/ALK Expression Analysis in Laryngeal Squamous Cell Carcinoma. In Vivo. 2019;33(3):815-819. https://doi.org/10.21873/invivo.11544

21. Tsiambas E, Alexopoulou D, Lambropoulou S, Gerontopoulos K, Karakitsos P, Karameris A. Targeting topoisomerase IIa in endometrial adenocarcinoma: a combined chromogenic in situ hybridization and immunohistochemistry study based on tissue microarrays. International Journal of Gynecological Cancer. 2006 05;16(3):1424-1431. https://doi.org/10.1111/ j.1525-1438.2006.00562.x

22. Tsiambas E, Manaios L, Papanikolopoulos C, Rigopoulos DN, Tsounis D, Karameris A, Soultati A, Koliopoulou A, Kravvaritis C, Sergentanis T, Patsouris E, Dourakis S. Chromogenic In Situ Hybridization Analysis of Epidermal Growth Factor Receptor Gene/Chromosome 7 Numerical Aberrations in Hepatocellular Carcinoma Based on Tissue Microarrays. Pathology \& Oncology Research. 200901 16;15(3):511-520. https://doi.org/10.1007/s12253-0089146-5

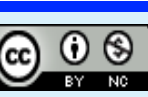

This work is licensed under a Creative Commons AttributionNon Commercial 4.0 International License. 